\title{
Wie Waldfachleute die Nachhaltigkeit der Wald- nutzung in der Schweiz einschätzen
}

\author{
Andreas Bernasconi \\ Peter Brang \\ Olivier Schneider
}

\author{
Pan Bern AG $(\mathrm{CH})^{*}$ \\ Eidgenössische Forschungsanstalt WSL, Waldressourcen und -management $(\mathrm{CH})$ \\ Bundesamt für Umwelt, Abteilung Wald (CH)
}

\section{How forestry professionals assess the status of sustainable forest management in Switzerland}

\begin{abstract}
The $170^{\text {th }}$ Annual Assembly of the Swiss Forestry Society took place at Andermatt in August 2013 with the theme "300 years of sustainable management". About one hundred forestry professionals took part in a seminar to debate the lessons of the last three centuries of sustainability for forest management. This article summarises the seminar results and draws conclusions for forest management in the future. The participants recognised that the greatest shortcomings were in the economic and social spheres. Measures were proposed in the area of knowledge of sustainable management, understanding of the forest ecosystem and in payment for forest services. The greatest challenge is considered to be responding to societal contradictions.
\end{abstract}

Keywords: sustainable forest management, forest policy, forest resources, Switzerland doi: $10.3188 /$ szf. 2014.0068

* Hirschengraben 24, Postfach 7511, CH-3001 Bern, E-Mail andreas.bernasconi@panbern.ch
$\mathrm{D}$ ie 170. Jahresversammlung des Schweizerischen Forstvereins, die am 22./23. August 2013 in Andermatt über die Bühne ging, stand unter dem Motto «300 Jahre Nachhaltigkeit». Dabei setzten sich die rund 100 anwesenden Waldfachleute im Rahmen eines Seminars mit der Frage auseinander, was aus drei Jahrhunderten Nachhaltigkeit im Wald für heute und für die Zukunft gelernt werden kann. Auslöser für das Seminarthema war das 300-Jahr-Jubiläum des Begriffes Nachhaltigkeit. Bei Hannß Carl von Carlowitz tauchte dieser Begriff 1713 erstmals auf: ... Wird derhalben die grösste Kunst, Wissenschaft, Fleiss und Einrichtung hiesiger Lande darinnen beruhen, wie eine sothane Conservation und Anbau des Holzes anzustellen, dass es eine continuierliche beständige und nachhaltende Nutzung gebe, weil es eine unentbehrliche Sache ist, ohne welche das Land in seinem Esse nicht bleiben mag (Carlowitz 1713: 105).

Im Jahr 1992, also vor gut 20 Jahren, einigten sich 170 Staaten im Rahmen der UNO-Konferenz für Umwelt und Entwicklung auf das Leitbild der nachhaltigen Entwicklung als Grundlage ihrer Politik. Die von der Brundtland-Kommission im Bericht «Our common future» (WECD 1987) formulierte Vision wurde zwar allgemein akzeptiert, aber unterschiedlich interpretiert und ausgelegt, und bei näherer Betrachtung werden zahlreiche Konflikte und
Defizite deutlich (vgl. Lambrecht \& Thierstein 1998 oder Birkmann et al 1999).

Vor diesem Hintergrund widmete sich das Seminar des Schweizerischen Forstvereins zwei Fragen, einerseits rückblickend: Welche Spuren haben der Begriff Nachhaltigkeit und sein Verständnis im Wald hinterlassen und welches waren die Erfolgsfaktoren? Andererseits vorausschauend: Wie sehen die Schweizer Waldfachleute die Nachhaltigkeit heute, und welches sind die damit verbundenen Herausforderungen für die zukünftige Bewirtschaftung der Wälder? Diese Fragen wurden zunächst in einer im Vorfeld der Jahresversammlung durchgeführten Umfrage thematisiert und anschliessend an der Jahresversammlung in einem Workshop vertieft (9 Gruppen mit je 6-11 Teilnehmenden, total 82 Teilnehmende). Letzterer wurde in Anlehnung an die SOAR-Methode - Strengths, Opportunities, Aspirations, Results strukturiert (Bruck \& Müller 2007) und dauerte 90 Minuten. Die Ergebnisse der Umfrage und des Workshops wurden im Nachgang zum Seminar analysiert und ausgewertet (Bernasconi 2013).

Ziel des vorliegenden Aufsatzes ist es, diese Ergebnisse zusammenzufassen und einem breiteren Publikum zugänglich zu machen sowie Denkanstösse zur Weiterentwicklung des forstlichen Nachhaltigkeitskonzeptes zu vermitteln. 
1 Erhaltung und Verbesserung

Waldfläche, Holzvorrat, Altersstruktur/

der Waldressourcen und ihres

Durchmesserverteilung, Kohlenstoffvorrat

Beitrags zum globalen Kohlen-

stoffkreislauf

2 Erhaltung der Gesundheit und Vitalität der Waldökosysteme

3 Erhaltung und Förderung der Nutzfunktionen der Wälder (Holz und Nichtholz)

4 Unterhalt, Schutz und Verbesserung der biologischen Vielfalt in Waldökosystemen

5 Erhaltung und Verbesserung der Schutzfunktion der Wälder (insbesondere Boden- und Wasserschutz)

6 Erhaltung anderer sozioökonomischer Funktionen

Ablagerung von Luftschadstoffen, Bodenzustand, Nadel-/Blattverlust, Waldschäden Holzzuwachs und -einschlag, Rundholz, Nichtholzprodukte, Dienstleistungen, Wälder mit Bewirtschaftungsplänen Natürlichkeitsgrad, eingebürgerte Baumschaftsmuster, gefährdete Waldarten, geschützte Wälder anderer Ökosystemfunktionen, Schutzwälteter natürlicher Ressourcen Bruttoinlandprodukt, Nettoerlös, Dienst-
Baumartenzusammensetzung, Verjüngung, arten, Totholz, genetische Ressourcen, Land-

Schutzwälder bezüglich Boden, Wasser und der bezüglich Infrastruktur und bewirtschaf-

Forstbetriebe, Beitrag des Waldsektors zum leistungsausgaben, Arbeitnehmer im Waldsektor, Sicherheit und Gesundheit am Arbeitsplatz, Holzverbrauch, Holzhandel, Holzenergie, Zutritt zu Erholungszwecken, kulturelle und spirituelle Werte

Tab 1 Überblick über die 6 Kriterien und 35 quantitativen, paneuropäischen Indikatoren einer nachhaltigen Waldbewirtschaftung gemäss Forest Europe et al (2011).

\section{Sustainable Forest Management}

Das französische Pendant zu «nachhaltig», «soutenu», ist bereits 1346 in der Ordonnance de Brunoy belegt: Les Mestres des forez ... ê̂ regart de ce que lesdites forez \& bois se puissent perpetuellement soustenir en bon estat (Le Tacon 2012: 84). Der heutige deutsche Begriff «nachhaltig» tauchte 1713 in der Form «nachhaltend» erstmals in der Literatur auf (Carlowitz 1713). Carlowitz warnte eindringlich davor, mehr Holz zu konsumieren, als der Wald zu erzeugen und tragen vermag. Er forderte bereits vor 300 Jahren, dass der Mensch mit der Natur agieren solle und nicht wider sie, und rief zur Verantwortung für die Mitwelt und die nachfolgenden Generationen auf. Dabei signalisiert «nachhaltend» ein aktives Handeln, das darauf abzielt, dass etwas erhalten bleibt (Sächsische Carlowitz-Gesellschaft 2013). Nachhaltigkeit zielt damit laut Grober (2013) immer auf die Bildung von Reserven, weil auf sofortige Nutzung zugunsten späterer Nutzungen und Nutzer verzichtet wird.

Die Einsicht in die Notwendigkeit, die Wirtschafts- und Lebensgrundlage für Kinder und Kindeskinder zu erhalten, ist mit Sicherheit jedoch viel älter und kann nach Radkau (2000) als selbstverständliche Norm der alten Bauernwirtschaft aufge- fasst werden; so selbstverständlich, dass es nicht einmal einen Begriff dafür gab (Detten \& Oesten 2013: $52)$.

Das heute international geltende forstfachliche Leitparadigma der Waldbewirtschaftung ist das Konzept des Sustainable Forest Management (SFM). Gemäss der Ministerkonferenz zum Schutz der Wälder in Europa (MCPFE, heute «Forest Europe») bedeutet SFM die Bewirtschaftung und Nutzung von Wäldern und Waldflächen auf eine Weise und in einem Rhythmus, welche die Erhaltung der biologischen Vielfalt, Produktivität, Verjüngungsfähigkeit und Vitalität des Waldökosystems auf Dauer gewährleisten und welche anderen Ökosystemen keine Schäden zufügen. Durch eine nachhaltige Bewirtschaftung können Wälder ihre ökologischen, ökonomischen und sozialen Funktionen auf nationaler und internationaler Ebene erfüllen. (BAFU 2013a: 175, in Anlehnung an MCPFE 2002).

MCPFE respektive Forest Europe hat den Rahmen für eine nachhaltige Waldbewirtschaftung mit 6 Kriterien und 35 quantitativen Indikatoren umfassend beschrieben (Tabelle 1). Dieser Rahmen wird kontinuierlich weiterentwickelt und angepasst (Forest Europe et al 2011). An ihm orientiert sich auch der Schweizer Waldbericht (BUWAL \& WSL 2005).

Das heutige, die Waldbewirtschaftungspraxis prägende Verständnis von SFM ist sehr breit angelegt. Auch die schweizerische Waldpolitik ist der Nachhaltigkeit im Sinne der internationalen Vereinbarungen verpflichtet und verfolgt zurzeit elf Ziele zur Sicherstellung der nachhaltigen Waldbewirtschaftung in der Schweiz (BAFU 2013b).

\section{Ist die Nachhaltigkeit der Wald- nutzung heute sichergestellt?}

Im Vorfeld des Seminars wurden alle 121 Personen, welche sich für die Jahresversammlung des SFV angemeldet hatten, schriftlich zur Einschätzung der Nachhaltigkeit der Waldnutzung in der Schweiz befragt. Diese Umfrage diente der Sensibilisierung für das Seminarthema, und deren Ergebnisse wurden als Einstieg in den Workshop genutzt. An der Umfrage nahmen insgesamt 88 Personen teil (Rücklaufquote 73\%). Die erste der drei gestellten Fragen lautete: «Ist die Nachhaltigkeit der Waldnutzung sichergestellt?» Die zweite Frage zielte auf die Begründung dieser Einschätzung, und die dritte befasste sich mit den Instrumenten, welche die Nachhaltigkeit der Waldnutzung sicherstellen sollen, sowie mit den Folgerungen für eine nachhaltige Waldbewirtschaftung.

Auf die Frage «Ist die Nachhaltigkeit der Waldnutzung sichergestellt?» antworteten 13\% der Teilnehmenden mit «vollständig», 50\% mit «grösstenteils», 36\% mit «teilweise» und 1\% mit «gar nicht». Als wichtigste Gründe für die Einschätzung, dass die 
Nachhaltigkeit vollständig erfüllt sei, wurden einerseits der allgemein gute Waldzustand in der Schweiz, welcher ein grosses Potenzial für verschiedene Waldnutzungen und damit für die Befriedigung von verschiedenen Bedürfnissen biete, und andererseits eine ausgezeichnete Forstorganisation sowie umfassende und gut verankerte rechtliche und planerische Grundlagen erwähnt.

Als wichtigste Gründe dafür, dass die Nachhaltigkeit nicht vollständig erfüllt sei, wurden ins Feld geführt: ungenügende Wirtschaftlichkeit der Waldbewirtschaftung, ungelöste Fragen im Umgang mit den gesellschaftlichen Ansprüchen an den Wald, Manko im Bereich der Artenvielfalt, ungenügende Holznutzung und Waldpflege sowie ungenügende Grundlagen, fehlende Strategien und fehlende Planung. Mit Abstand am häufigsten wurden Argumente im Zusammenhang mit der ungenügenden Wirtschaftlichkeit genannt.

Auch in den Gruppenarbeiten am Seminar wurde einleitend die Frage nach der Nachhaltigkeit der Waldnutzung gestellt. Die Einschätzungen waren sehr ähnlich wie in der Umfrage vor dem Seminar. Zusätzlich wurden als Gründe dafür, dass die Nachhaltigkeit erfüllt sei, der Waldaufbau, das hohe
Mass der Gewährleistung der Waldfunktionen und damit verbunden eine hohe Zufriedenheit der Bevölkerung mit dem Wald und seiner Bewirtschaftung genannt. Insgesamt wird die Situation in der Schweiz als - im internationalen Vergleich - vorbildlich erachtet. Als zusätzliche Gründe für eine nicht erfüllte Nachhaltigkeit wurden insbesondere gesellschaftliche Veränderungen, der damit verbundene Druck auf den Wald (Siedlungsentwicklung, zunehmende Erholungsnutzung im urbanen Raum) sowie die Auswirkungen der globalen sozioökonomischen Entwicklungen genannt.

Zusammenfassend waren die am Seminar teilnehmenden Waldfachleute der Meinung, dass die Nachhaltigkeit der Waldnutzung in der Schweiz im internationalen Vergleich gut gewährleistet ist. Es gibt jedoch mehrere Bereiche mit Verbesserungsbedarf.

\section{Handlungsbedarf und Massnahmen}

Gestützt auf die Analyse der Ausgangslage (Waldnutzung) ermittelten die neun Gruppen den Handlungsbedarf (Waldbewirtschaftung), und sie

\begin{tabular}{|c|c|c|c|}
\hline \multirow{2}{*}{\multicolumn{2}{|c|}{ Waldpolitik 2020}} & \multicolumn{2}{|c|}{ Am Seminar erkannter Handlungsbedarf } \\
\hline & & $\begin{array}{l}\text { Anzahl Nennungen } \\
\text { (total 74) }\end{array}$ & Wichtigste Aspekte \\
\hline \multicolumn{4}{|c|}{ Schwerpunktziele } \\
\hline 2 & $\begin{array}{l}\text { Klimawandel: Minderung und Anpassung ist } \\
\text { sichergestellt }\end{array}$ & 3 & Jungwaldpflege \\
\hline 4 & $\begin{array}{l}\text { Die Biodiversität bleibt erhalten und ist ge- } \\
\text { zielt verbessert }\end{array}$ & 3 & Defizite im Bereich der Biodiversität \\
\hline 5 & Die Waldfläche bleibt erhalten & 0 & $\begin{array}{l}\text { In den Gruppen wurde zu diesem Ziel kein spezifischer Handlungsbedarf } \\
\text { angemeldet }\end{array}$ \\
\hline 7 & $\begin{array}{l}\text { Die Waldböden, das Trinkwasser und die Vita- } \\
\text { lität der Bäume sind nicht gefährdet }\end{array}$ & 0 & $\begin{array}{l}\text { In den Gruppen wurde zu diesem Ziel kein spezifischer Handlungsbedarf } \\
\text { angemeldet }\end{array}$ \\
\hline 8 & $\begin{array}{l}\text { Der Wald wird vor Schadorganismen } \\
\text { geschützt }\end{array}$ & 0 & $\begin{array}{l}\text { In den Gruppen wurde zu diesem Ziel kein spezifischer Handlungsbedarf } \\
\text { angemeldet }\end{array}$ \\
\hline 9 & Wald und Wild stehen in einem Gleichgewicht & 2 & Wald-Wild-Problematik, Kontinuität der Verjüngung \\
\hline 10 & $\begin{array}{l}\text { Die Freizeit- und Erholungsnutzung erfolgt } \\
\text { schonend }\end{array}$ & 4 & $\begin{array}{l}\text { Schonende Erholungsnutzung, Umgang mit dem Erholungsdruck, } \\
\text { Sicherstellung des freien Betretungsrechtes, Abfallproblematik }\end{array}$ \\
\hline 11 & $\begin{array}{l}\text { Bildung, Forschung und Wissenstransfer sind } \\
\text { gewährleistet }\end{array}$ & 32 & $\begin{array}{l}\text { Wissenssicherung, hochstehende Ausbildung der Waldfachleute, } \\
\text { kontinuierlicher Wissenstransfer zwischen Forschung und Praxis und } \\
\text { umgekehrt, Nachhaltigkeitsverständnis in der Bevölkerung }\end{array}$ \\
\hline
\end{tabular}

Tab 2 Am Seminar bezeichneter Handlungsbedarf und Ex-post-Zuordnung der vorgebrachten Argumente zu den Zielen der Waldpolitik 2020 (BAFU $2013 b$ ). 


\begin{tabular}{|l|c|c|c|}
\hline Handlungsfeld & $\begin{array}{c}\text { Anzahl } \\
\text { Massnahmen }\end{array}$ & $\begin{array}{c}\text { Gewichtung des } \\
\text { Handlungsfelds }\end{array}$ & $\begin{array}{c}\text { Anteile der } \\
\text { Gewichte }\end{array}$ \\
\hline $\begin{array}{l}\text { Gesellschaftlicher Dialog } \\
\text { und Bildung }\end{array}$ & 28 & 79 & $38 \%$ \\
\hline $\begin{array}{l}\text { Inwertsetzung von } \\
\text { Waldleistungen }\end{array}$ & 12 & 58 & $28 \%$ \\
\hline Governance & 17 & 49 & $23 \%$ \\
\hline Ökosystemmanagement & 7 & 22 & $11 \%$ \\
\hline Total & 64 & 208 & $100 \%$ \\
\hline
\end{tabular}

Tab 3 Zuordnung der vorgeschlagenen Massnahmen zu vier Handlungsfeldern. Dargestellt sind die Anzahl Massnahmen pro Handlungsfeld und die Gewichtung der Handlungsfelder.

erarbeiteten einen Massnahmenkatalog. Zum Handlungsbedarf gab es insgesamt 74 Nennungen, die wir nach dem Seminar den Zielen der Waldpolitik 2020 (BAFU 2013b) zuordneten. Dabei zeigte sich, dass rund 43\% der eingebrachten Argumente das Ziel «Bildung, Forschung und Wissenstransfer» betreffen. Von diesen $43 \%$ steht wiederum der Grossteil der Massnahmen im Zusammenhang mit der Sensibilisierung und der Bewusstseinsbildung der gesellschaftlichen Akteursgruppen (Tabelle 2).

Im letzten Schritt wurden ausgehend vom Handlungsbedarf Massnahmen zur langfristigen Sicherstellung einer nachhaltigen Waldbewirtschaftung zusammengetragen. Die Gruppen umschrieben je vier bis neun Massnahmen. Die Teilnehmenden gewichteten dann die Massnahmen ihrer eigenen Gruppe (je gleich viele Punkte pro Person). Nachträglich wurden die insgesamt 64 Massnahmen folgenden vier Handlungsfeldern zugewiesen: 1) Gesellschaftlicher Dialog und Bildung, 2) Inwertsetzung von Waldleistungen, 3) Governance und 4) Ökosystemmanagement.

Dabei zeigte sich, dass am meisten Massnahmen im Handlungsfeld «gesellschaftlicher Dialog und Bildung» angesiedelt werden können (Tabelle 3) und dass diese Massnahmen als am bedeutendsten angesehen wurden. Der wirksamste Hebel zur Behebung der grössten erkannten Mängel liegt somit - nach Meinung der Seminarteilnehmenden nicht dort, wo die Mängel auftreten, sondern im gesellschaftlichen und politischen Umfeld. Als am zweitwichtigsten wurden Massnahmen zur Inwertsetzung von Waldleistungen beurteilt (Tabelle 3).

\section{Gleichzeitig in vier Handlungsfeldern wirken}

Mit einer Rücklaufquote der schriftlichen Umfrage von 73\% war das Interesse der Befragten am diskutierten Thema hoch, und auch die Gespräche in den Gruppen verliefen sehr angeregt. Die Diskussion war intensiv, aber angesichts der knappen Zeit recht spontan und stark durch die persönliche Sichtweise der Anwesenden - hauptsächlich Mitglieder des Schweizerischen Forstvereins - geprägt. Trotzdem ergaben sich eindeutige und in allen Gruppen besprochene Schwerpunkte wie etwa ein erhebliches Defizit bei wirtschaftlichen Aspekten sowie eine durchwegs hohe Gewichtung der vorgeschlagenen Massnahmen im Bereich des gesellschaftlichen Dialogs und der Bildung.

Das Seminar gab einen guten Überblick darüber, wie die anwesenden Waldfachleute die Nachhaltigkeit der Waldnutzung heute beurteilen und in welchen Bereichen sie die grössten Herausforderungen sehen. Die Nachhaltigkeit ist für sie ein wichtiges und aktuelles Leitthema. Ähnliches war bereits 1993 festzustellen, als sich der Schweizerische Forstverein anlässlich seines 150-Jahr-Jubiläums mit der Nachhaltigkeit auseinandersetzte (Schuler 1995). Pfister (1995: 134) fasste die Ergebnisse damals mit folgenden Worten zusammen: Hinsichtlich des Nachhaltigkeitsgedankens wurde offensichtlich, dass dieser an Bedeutung und Aktualität nichts eingebüsst hat. Er muss im Gegenteil in den kommenden Jahrzehnten noch viel mehr bewusst gemacht werden. Dabei müssen die Forstleute Pioniere bleiben, um die zeitgemässe Ausprägung der Nachhaltigkeit in der Waldbewirtschaftung ringen, sich aber auch dem gesellschaftlichen Umfeld öffnen und Impulse zur Lösung der Probleme ebenfalls in nicht forstlichen Bereichen geben.

20 Jahre später zielen die Schlussfolgerungen in eine ähnliche Richtung. Die Diskussionen anlässlich des Seminars zeigen, dass trotz neuen Konzepten wie etwa demjenigen der "grünen Wirtschaft» die Nachhaltigkeit auch in Zukunft ein prägender Leitgedanke für die forstlichen Akteure bleiben wird, und sie unterstreichen die Bedeutung des gesellschaftlichen Dialogs.

Die Rio-Deklaration von 1992 hat nachhaltige Entwicklung als eine Trias von Wirtschaft, Gesellschaft und Umwelt definiert, welche mit dem Bild eines gleichseitigen Dreiecks oder von drei Säulen veranschaulicht wird. Auch auf Bundesebene ist die nachhaltige Entwicklung im Sinne eines erweiterten Dreidimensionenmodells definiert (Wachter 2012; Wachter 2014, dieses Heft). Diese drei Dimensionen sollen gleichrangig entwickelt werden. Das überzeugende Grundkonzept lässt jedoch in der Umsetzung viele Fragen offen, weil hinter den drei Dimensionen unterschiedliche und teilweise konkurrierende Interessen stehen (Sächsische CarlowitzGesellschaft 2013).

In Abbildung 1 sind die oben beschriebenen vier Handlungsfelder in das Nachhaltigkeitsdreieck projiziert und mit Beispielen illustriert. In allen vier Handlungsfeldern sahen die Seminarteilnehmenden Handlungsbedarf, und sie identifizierten Massnahmen. Nachhaltigkeit betrifft stets alle vier Handlungsfelder und bedingt die Gleichzeitigkeit des Handelns in diesen vier Handlungsfeldern. Anders gesagt: Die Maximierung von Massnahmen in ei- 


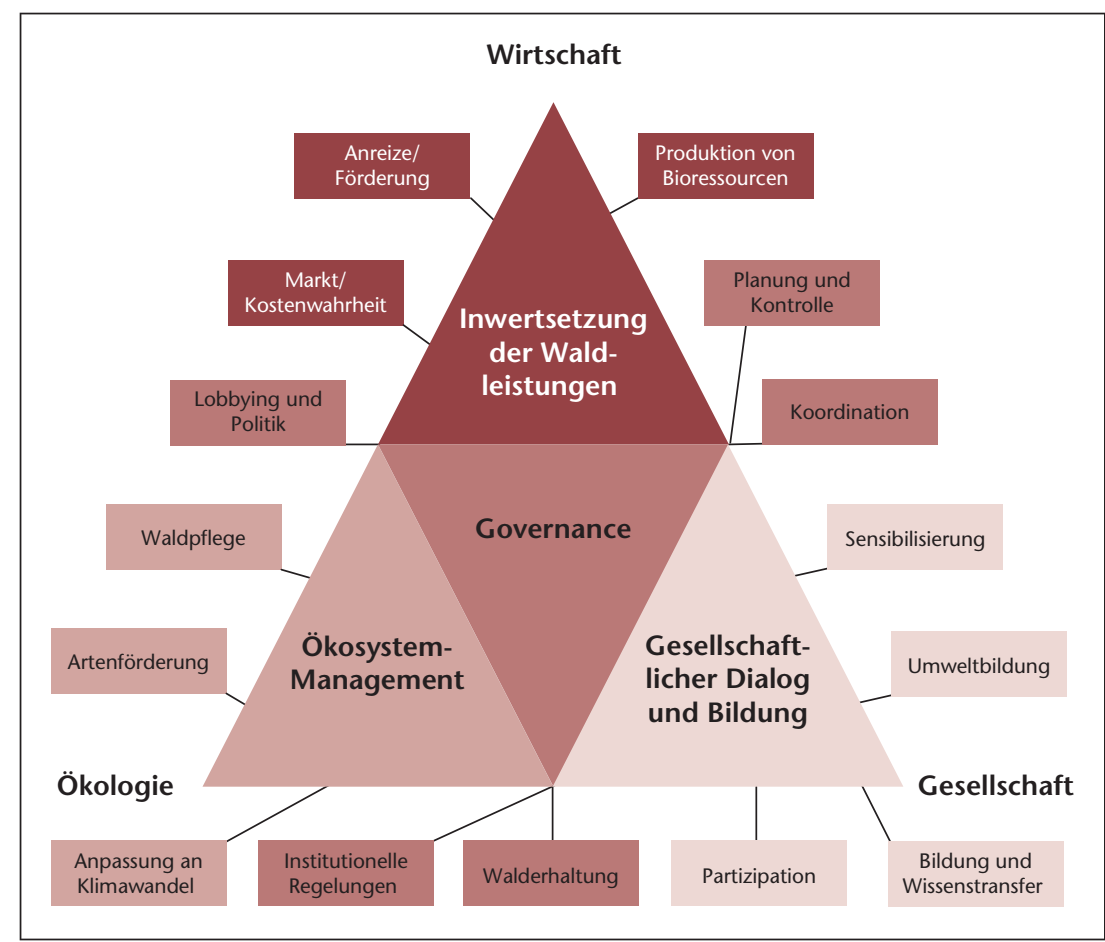

Abb 1 Einbettung der vier Handlungsfelder in das Nachhaltigkeitsdreieck und Beispielaspekte der Handlungsfelder (Rechtecke).

nem Handlungsfeld zulasten eines anderen ist nicht zielführend.

Die Seminarteilnehmenden - die auch als weitgehend repräsentative Stichprobe der Mitglieder des Forstvereins betrachtet werden können - sahen insgesamt Defizite in allen vier Handlungsfeldern und vertraten - zumindest im Kollektiv ihrer Gruppe - eine integrale Sichtweise. Die grössten aktuellen Defizite werden im ökonomischen und gesellschaftlichen Bereich angesiedelt, entsprechend sind der primäre Handlungsbedarf und die zu treffenden Massnahmen beim Nachhaltigkeitswissen, beim Waldverständnis und bei der Inwertsetzung der Waldleistungen verortet. Als grosse Herausforderung wird dabei der Umgang mit den gesellschaftlichen Widersprüchen angesehen. Diese Sichtweise dürfte stark geprägt sein vom beruflichen Hintergrund der Teilnehmenden, von denen viele in Behörden und diesen nahestehenden Institutionen tätig sind.

\section{Zum Schluss}

Das Leitbild der nachhaltigen Entwicklung erfordert eine sorgfältige Operationalisierung. Mit der Suche nach einer abschliessenden Theorie würde der falsche Weg eingeschlagen (Birkmann et al 1999). Die UNO-Konferenz Rio+20 hat im Sommer 2012 das Bekenntnis der Weltgemeinschaft zur Nachhaltigkeit bekräftigt. Die Klärung und Schärfung des Konzepts bleibt eine Daueraufgabe.

Die bisherigen Modelle zur Nachhaltigkeit enthalten kaum Lösungsansätze, um mit den inhä- renten Interessengegensätzen und Widersprüchen umzugehen. Voraussetzung zur Überwindung dieser Herausforderung ist ein fortwährender Prozess zur Aushandlung der Ziele und der Umsetzungsschwerpunkte. Detten \& Oesten (2013: 56) bezeichnen den Begriff Nachhaltigkeit als «Grenzkonzept»: Der Begriff ist gemeinsamer Ansatzpunkt unterschiedlichster Nachhaltigkeitsverständnisse, und seine Verwendung wird zum fortwährenden Anlass zu einer transparenteren, unterschiedliche Werthaltungen beleuchtenden Diskussion über verschiedene «nachhaltigkeitsbezogene» Optionen. Aufgrund dessen folgern sie, dass der Illusion des Langfristwissens beziehungsweise der Langfristplanung paradoxerweise eine Absage erteilt werden müsse, die langfristige Sichtweise aber keinesfalls aufgegeben werden dürfe.

Die Kunst wird somit darin bestehen, die massgebenden Interessengruppen in einen auf die Langfristperspektive ausgerichteten, permanenten Dialog einzubinden, dies im Wissen um die Unsicherheit darüber, wie sich die gesellschaftlichen Bedürfnisse und das Klima entwickeln werden, in Kenntnis der vorhandenen multiplen Rationalitäten (Schedler \& Rüegg-Stürm 2013) und unter der Bedingung, dass keine der drei Nachhaltigkeitsdimensionen zulasten der anderen maximiert werden darf. Ein Beispiel für einen derartigen Aushandlungsprozess ist das Positionspapier «Biodiversität und Holzproduktion unter einem Dach» des Schweizerischen Forstvereins (SFV 2013). Der Forstverein wird sich mit den anlässlich des Seminars von seinen Mitgliedern aufgeworfenen Fragen und vorgeschlagenen Massnahmen im Rahmen der Umsetzung seiner waldpolitischen Agenda weiter auseinandersetzen.

Eingereicht: 18. Dezember 2013, akzeptiert (ohne Review): 24. Januar 2014

\section{Literatur}

BAFU (2013A) Jahrbuch Wald und Holz 2013. Bern: Bundesamt Umwelt. 180 p.

BAFU (2013B) Waldpolitik 2020. Visionen, Ziele und Massnahmen für eine nachhaltige Bewirtschaftung des Schweizer Waldes. Bern: Bundesamt Umwelt. 66 p.

BERNASCONI A (2013) 300 Jahre Nachhaltigkeit - Jahresversammlung des Schweizerischen Forstvereins. Grundlagen und Auswertung des Workshops vom 22. August 2013 in Andermatt. Bern: Pan Bern. 23 p.

BIRKMANN J, KOITKA H, KREIBICH V, LIENENKAMP R (1999) Indikatoren für eine nachhaltige Raumentwicklung. Methoden und Konzepte der Indikatorenforschung. Dortmund: Techn Univ, Dortmunder Beiträge zur Raumplanung 96. 173 p.

BRUCK W, MÜLLER R (2007) Wirkungsvolle Tagungen und Grossgruppen. Ziele, Wirkfaktoren und Designs. Offenbach: Gabal. $434 \mathrm{p}$.

BUWAL, WSL, EDITORS (2005) Waldbericht 2005. Zahlen und Fakten zum Zustand des Schweizer Waldes. Bern: Bundesamt Umwelt Wald Landsch. $150 \mathrm{p}$.

CARLOWITZ HC VON (1713) Sylvicultura oeconomica oder Hausswirthliche Nachricht und Naturmässige Anweisung zur Wilden Baum-Zucht. Leipzig: Johann Friedrich Braun. 
DETTEN R VON, OESTEN G (2013) Nachhaltige Waldwirtschaft ein Modell für nachhaltige Entwicklung? Nat Landsch 88 (2): 52-57.

FOREST EUROPE, UNECE, FAO (2011) State of Europe's forests 2011. Status and trends in sustainable forest management in Europe. Aas: Forest Europe. 337 p.

GROBER U (2013) Urtexte - Carlowitz und die Quellen unseres Nachhaltigkeitsbegriffs. Nat Landsch 88 (2): 46-51.

LAMBRECHT M, THIERSTEIN A (1998) Nachhaltige Entwicklung und Raumordnung. Ein methodisches Konzept für die Zusammenführung globaler Ziele und nationaler Politikfelder am Beispiel der schweizerischen Raumordnungspolitik. Raumforsch Raumordn 56: 101-110.

LE TACON F (2012) Développement durable ou gestion durable? Revue Forestière Française LXIV (1): 83-96.

MCPFE (2002) Improved Pan-European Indicators for Sustainable Forest Management. Vienna: Ministerial Conference Protection Forests Europe. 6 p. www.foresteurope.org/docs/reporting/Vienna_Improved_Indicators.pdf (9.1.2014)

PFISTER F (1995) Nachhaltigkeit als Postulat der Forstwirtschaft gerechtfertigt, haltbar, übertragbar? Schweiz Z Forstwes Beih 75: 129-134.

\section{Wie Waldfachleute die Nachhaltigkeit der Waldnutzung in der Schweiz einschätzen}

Die 170. Jahresversammlung des Schweizerischen Forstvereins vom August 2013 in Andermatt stand unter dem Motto «300 Jahre Nachhaltigkeit». Die rund 100 anwesenden Waldfachleute setzten sich im Rahmen eines Seminars mit der Frage auseinander, was aus drei Jahrhunderten Nachhaltigkeit im Wald für heute und für die Zukunft gelernt werden kann. Im vorliegenden Aufsatz werden die Workshop-Ergebnisse zusammengefasst und Folgerungen für die künftige Waldbewirtschaftung gezogen. Die Seminarteilnehmenden erkannten die grössten aktuellen Defizite im ökonomischen und im gesellschaftlichen Bereich. Massnahmen werden in den Bereichen Nachhaltigkeitswissen, Waldverständnis und bei der Inwertsetzung der Waldleistungen vorgeschlagen. Als grosse Herausforderung wird der Umgang mit gesellschaftlichen Widersprüchen angesehen.
RADKAU J (2000) Natur und Macht. Eine Weltgeschichte der Umwelt. München: Beck. 438 p.

SÄCHSISCHE CARLOWITZ-GESELLSCHAFT, EDITOR (2013) Die Erfindung der Nachhaltigkeit. Leben, Werk und Wirkung des Hans Carl von Carlowitz. München: Oekom. 285 p.

SCHEDLER K, RÜEGG-STÜRM J (2013) Multirationales Management. Der erfolgreiche Umgang mit widersprüchlichen Anforderungen an die Organisation. Bern: Haupt. 244 p.

SCHULER A, EDITOR (1995) Nachhaltigkeit als Grundlage menschlichen Handelns. Seminar anlässlich des 150-Jahr-Jubiläums des Schweizerischen Forstvereins vom 8.-11. September 1993 in Interlaken. Schweiz Z Forstwes Beih 75. 146 p.

SFV (2013) Biodiversität und Holzproduktion unter einem Dach. Positionspapier. Schweiz Z Forstwes 164: 198-205.

SCHWEIZERISCHER BUNDESRAT (2012) Strategie Nachhaltige Entwicklung 2012-2015. Bern: Schweizerischer Bundesrat. 72 p. WACHTER D (2012) Nachhaltige Entwicklung. Das Konzept und seine Umsetzung in der Schweiz. Chur: Rügger, 3 ed. 188 p.

WACHTER D (2014) Stand der Nachhaltigkeitsdiskussion - Denkmodelle der Nachhaltigkeit. Schweiz Z Forstwes 165: 53-60. doi: $10.3188 / 2014.0053$

WCED (1987) Our common future. Oxford: Oxford Univ Press. $400 \mathrm{p}$.

\section{Comment les professionnels de la forêt jugent la situation de la gestion durable de la forêt en Suisse}

La 170e Assemblée annuelle de la Société forestière suisse s'est tenue à Andermatt en août 2013 sur le thème de «300 ans de gestion durable». Les quelque 100 professionnels de la forêt ont débattu lors d'un séminaire des enseignements de ces trois siècles de durabilité pour la gestion actuelle et future de la forêt. Le présent article résume les résultats du séminaire et en tire des conclusions pour la gestion forestière future. Les participants ont reconnu que les plus grandes déficiences se situent aux niveaux économique et social. Des mesures ont été proposées dans les domaines de la connaissance de la gestion durable, la compréhension de l'écosystème forestier et dans la valorisation des prestations de la forêt. La réponse aux contradictions sociétales est considérée comme le plus grand défi. 